Claudine Dietz

Sophie H.N. Swinkels

Jan K. Buitelaar

Emma van Daalen

Herman van Engeland

Accepted: 6 February 2007

Published online: 30 March 2007

C. Dietz $(\bowtie) \cdot$ E. van Daalen

H. van Engeland

Dept. of Psychiatry, Rudolf Magnus

Institute of Neuroscience

University Medical Center Utrecht

P.O. 85500

3508 GA Utrecht, The Netherlands

E-Mail: cdietz@umcutrecht.nl

S.H.N. Swinkels · J.K. Buitelaar

Dept. of Psychiatry

Radboud University Nijmegen Medical

Center

Nijmegen, The Netherlands

S.H.N. Swinkels · J.K. Buitelaar

Dept. of Psychiatry

Karakter Child and Adolescent Psychiatry

University Center Nijmegen

Nijmegen, The Netherlands

\title{
Stability and change of IQ scores in preschool children diagnosed with autistic spectrum disorder
}

Abstract Aim To investigate cognitive development in preschool-age children diagnosed with Autistic Spectrum Disorder (ASD; $N=39$ ) compared with that of children diagnosed with mental retardation (MR; $N=14)$ and normally developing children (NC; $N=36$ ). Method In a prospective longitudinal study, cognitive development was tested at age 24 months (T1; SD $=6$ months) and 43 months (T2; SD = 5). Results Group IQ scores were stable between $\mathrm{T} 1$ and $\mathrm{T} 2$ as evidenced by high correlations $(r=.81$, $P<.01)$ and consistency of average group scores. At the same time however, about a third of children with ASD showed an increase of cognitive scores of 15 points or more. This increase of IQ was correlated with lower scores at the early screening of autistic traits (ESAT) at T1, higher IQ level at T2 and higher expressive language skills at T2. Intensity of treatment was not related to IQ increase. Conclusions High correlations between cognitive scores in preschool children with ASD suggest that measurements of cognitive function are valid at this age. We found indications of both stability and change of IQ scores. Findings suggest that some children with ASD show catch-up intellectual development. To the best of our knowledge, this increase in IQ scores cannot be attributed to treatment effects.

Key words IQ - intellectual development - preschool period - autism

\section{Introduction}

As a result of screening programs and greater public awareness, Autistic Spectrum Disorders (ASDs) are being diagnosed earlier in recent years [8]. Many children are now diagnosed in the preschool period, although diagnosis tends to be later if there is no intellectual disability [10]. While the early clinical diagnosis of autism appears to be stable [4, 12, 14], this is less clear for the stability of IQ and for the prognostic value of early cognitive testing.
Evaluation of cognitive strengths and weaknesses forms an essential part of clinical examination. Knowledge of a child's cognitive status can facilitate the planning of education and / or interventions and provide information about the child's prognosis. The prognostic value of cognitive tests in children with ASD has been demonstrated in several studies. For example, Nordin and Gillberg [24] found an IQ below 50 of about school age to predict severe restrictions in social and adaptive functioning in adult life. The absence of communicative speech at age 5-6 was found to be indicative of poor long-term outcome. McGov- 
ern and Sigman [20] found IQ to be stable between school age and adulthood in most children, but found IQ to increase especially in children who were high functioning at the initial measurement.

However, the value of cognitive testing at an early age in children diagnosed with autism may be compromised by at least two factors. First, the abilities tested at very early age are different from those measured at school age or adulthood [25]. Developmental tests for preschoolers tap sensory and motor capacities that cannot easily be compared with those of measures of later intelligence (e.g. conceptual thinking). Second, these tests have usually not been developed for and validated with children with developmental disabilities [23]. The more irregular pattern of cognitive abilities that typically characterizes clinical groups such as children with ASD, makes it important to choose an instrument that taps similar abilities at all measurement times (f.e. more verbal / non-verbal tests). Moreover, it may be difficult to test autistic children because of their limited attention skills or limited communicative and social abilities. Despite these problems in the early testing of autistic children, strong correlations have been demonstrated between IQ measured at age 2 and 4 years [32], although unstable cognitive scores have also been reported in children diagnosed with ASD. For example, IQ has been reported to decrease between the ages of 2 and 18 years [9] whereas others have shown cognitive scores to increase between 3 and 15 years in a cross-sectional study [19] and between 2 and 13 years in a longitudinal study [28]. Eaves and Ho [7] found IQ to increase and decrease between age $2^{1 / 2}$ and $4 \frac{1}{2}$, in their sample of 49 children diagnosed with ASD. They found that higher functioning children were most likely to show an increase in IQ score.

As part of a population screening study for ASD [5], we measured the cognitive abilities of a large group of very young children with ASD twice. The first assessment (T1) was carried out when the children were on average 24 months $(\mathrm{SD}=6)$ and the second (T2) when the children were on average 43 months $(\mathrm{SD}=6)$. We included a comparison group of children diagnosed with mental retardation (MR) without ASD and normal controls (NC).

The objectives of this study were the following: (1) To determine the stability of cognitive scores of children diagnosed with ASD between T1 and T2. Stability was measured in terms of correlations of cognitive scores and consistency of average group scores, and was compared to stability of cognitive scores in children diagnosed with MR and NC. (2) To explore individual changes of cognitive scores, and (3) To identify factors associated with cognitive change.

\section{Methods}

\section{Procedure}

Children were recruited by means of either a population screen or case finding (surveillance). The first group consisted of 31.724 children aged 14 to 15 months that were screened at well-baby clinics within a geographically defined area (Utrecht, The Netherlands). Screen-positive children were investigated during a home visit with screen II. This group of children is described in our earlier report [5]. The second group consisted of children up to age 36 months that were identified by surveillance by physicians of well-baby clinics because of concerns about their social development. These children were immediately investigated with screen II. Screen-positive children at screen II were evaluated at the Department of Child and Adolescent Psychiatry, University Medical Center Utrecht (Netherlands). Children were re-evaluated when they were about 42 months $(M=45.1, \mathrm{SD}=6.2)$ and clinical diagnoses were established.

Recruitment and testing of the children began in January 1999 and ended in April 2004. The Medical Ethics Review Board of the University Medical Center Utrecht approved the study design and screening procedure.

\section{Participants}

Thirty-nine children diagnosed with ASD were selected. Of these children, 19 (49\%) were low functioning at follow up measurement (IQ $<70$ ). As comparison groups, 14 children diagnosed with mental retardation without ASD were selected (MR) and a control group of 36 children (NC). The control group consisted of 12 typically developing children who were recruited at random from well-baby clinics, and 24 children who scored negative at screen II and who had scores below the clinical range at the Child Behavior Check List (CBCL) [1] and the Social Communication Questionnaire (SCQ) [3]. Children were on average 24 months $(\mathrm{SD}=6)$ at $\mathrm{T} 1$ and 43 months $(\mathrm{SD}=5)$ at $\mathrm{T} 2$. There were more boys than girls (66 versus 34\%). Descriptive information can be found in Table 1.

\section{Clinical measurements}

Screening was done with the Early Screening of Autistic Traits (ESAT) [30], the items of which refer to social development and play behavior. At screen I, the 4-item ESAT was used and completed by parents. 
Table 1 Descriptive information and cognitive scores for the clinical groups ASD (Autistic Spectrum Disorder), MR (Mental Retardation) and NC (Normal Controls at $\mathrm{T} 1$ and $\mathrm{T} 2$

\begin{tabular}{|c|c|c|c|}
\hline & $\operatorname{ASD}(N=14)$ & MR $(N=39)$ & $N C(N=36)$ \\
\hline Sex (\% boys) & 82 & 79 & 44 \\
\hline Mean age $\mathrm{T} 1$ (SD) & $25.15(8.04)$ & $25(4.64)$ & $22.81(4.76)$ \\
\hline Mean age $\mathrm{T} 2$ (SD) & $43.26(5.94)$ & $42.36(6.13)$ & $42.94(4.08)$ \\
\hline IQ T1 & $65(18.33)$ & $56.65(7.83)$ & $104.61(12.68)$ \\
\hline IQ T2 & $71.78(28.40)$ & $54.08(11.55)$ & $104.81(11.96)$ \\
\hline Total IQ change & $6.78(21.55) n s$ & $-2.57(11.39) n s$ & $-0.19(10.63) n s$ \\
\hline T-score verbal tests $T 1^{\mathrm{a}}$ & $33.13(9.82)$ & $24.72(6.41)$ & $51.61(8.70)$ \\
\hline$T$-score verbal tests $T 2^{\mathrm{a}}$ & $42.33(11.49)$ & $26.72(6.31)$ & $51.79(7.58)$ \\
\hline Change $T$-score verbal tests ${ }^{\mathrm{a}}$ & $9.20(10.60) * *$ & $-2(7.09) n s$ & $-0.18(7.28) n s$ \\
\hline T-score non-verbal tests $\mathrm{T}^{\mathrm{a}}$ & $35.98(12.02)$ & $25.33(7.13)$ & $52.74(6.37)$ \\
\hline T-score non-verbal tests $\mathrm{T}^{\mathrm{a}}$ & $41.93(11.35)$ & $26.72(6.31)$ & $51.79(7.58)$ \\
\hline Change $T$-score non-verbal tests ${ }^{\mathrm{a}}$ & $8.45(11.98)^{* *}$ & $-1.39(8.07) \mathrm{ns}$ & $0.94(7.71) \mathrm{ns}$ \\
\hline
\end{tabular}

ASD (autistic spectrum disorder); MR (mental retardation); NC (normal controls)

${ }^{a}$ Scores were calculated for a subgroup of children that were measured with the MSEL: ASD, $N=21 ; M R, N=9$; NC, $N=36$

** $P$-value $\leq .001$, ns $P$-value is not significant.

Children were considered screen-positive if at least 1 of 4 items was scored positively. At screen II, the 14item ESAT was completed by both parents and experts. Children were considered screen-positive if 3 of 14 items were scored positively.

Children that scored positive at screen II were assessed at T1 and T2 at the Department of Child and Adolescent Psychiatry of the UMC Utrecht. Assessments included developmental history, a standardized parental interview with the Autism Diagnostic Interview Revised (ADI-R) [13], the Vineland SocialEmotional Early Childhood Scales [29]; standardized behavior observation using the Autism Diagnostic Observation Schedule Generic or ADOS-G [6], and pediatric examination and medical work-up. The Child Behavior Checklist (CBCL 11/2-5) [1] was completed at T2 as well as the Social Communication Questionnaire (SCQ) [3].

Using this information, an experienced child psychiatrist (EvD, HvE, JB) formed a clinical diagnosis; the final diagnosis was established according to DSMIV criteria [2] at T2. The inter-rater reliability of the clinical diagnosis was calculated for two diagnostic categories namely ASD or other than ASD, for a subgroup of 38 children. Agreement was reached in $92 \%$ of 38 cases. Agreement corrected for chance was .74 (Cohen's Kappa). Diagnostic discrepancies were resolved at a consensus meeting.

Many children were treated between $\mathrm{T} 1$ and $\mathrm{T} 2$. We distinguished three categories: no treatment, low-intensity outpatient treatment (approximately $2 \mathrm{~h}$ a week at most), or high-intensity treatment (day care at least 2 days a week at a specialized psychiatric and/or educational center). Seventy-four percent of the children with ASD received high-intensity treatment, as opposed to $57 \%$ of the children with MR.

\section{Cognitive measures}

Most children were evaluated with the Mullen Scales of Early Learning (MSEL) [22]. The MSEL is an individually administered comprehensive measure of cognitive functioning of infants or preschool children aged up to 68 months. The MSEL consists of five scales, one measuring gross motor development and four cognitive scales (Visual Reception, Fine Motor functioning, Receptive Language, and Expressive Language). A composite score can be derived from the standardized $\mathrm{T}$ scores for the four cognitive scales. We used the four cognitive scales only. As Dutch normative data were not available, we used American normative data. The MSEL has excellent reliability and validity, as demonstrated in groups of children at risk of developmental delay [31]. Test-retest reliability measured within 2 weeks is high, particularly for children younger than 24 months [22].

Children that were low functioning or who were difficult to examine with the MSEL, were tested with the Psycho-educational Profile Revised (PEP-R) [26], as this instrument requires fewer task taking skills; 8 children at T1 (6 with ASD and 2 with MR) and 16 children at T2 (ASD, $N=11 ; \mathrm{MR}, N=5$ ). More children were tested with this instrument at T2 than at T1 because disruptive behavior was now either more common or interfered more with the tasks.

\section{Results}

\section{IQ stability measured by correlations between cognitive scores at $\mathrm{T} 1$ and $\mathrm{T} 2$}

In the total group of children, the correlation between IQ scores measured at T1 and T2 was high $(r=.81$, 


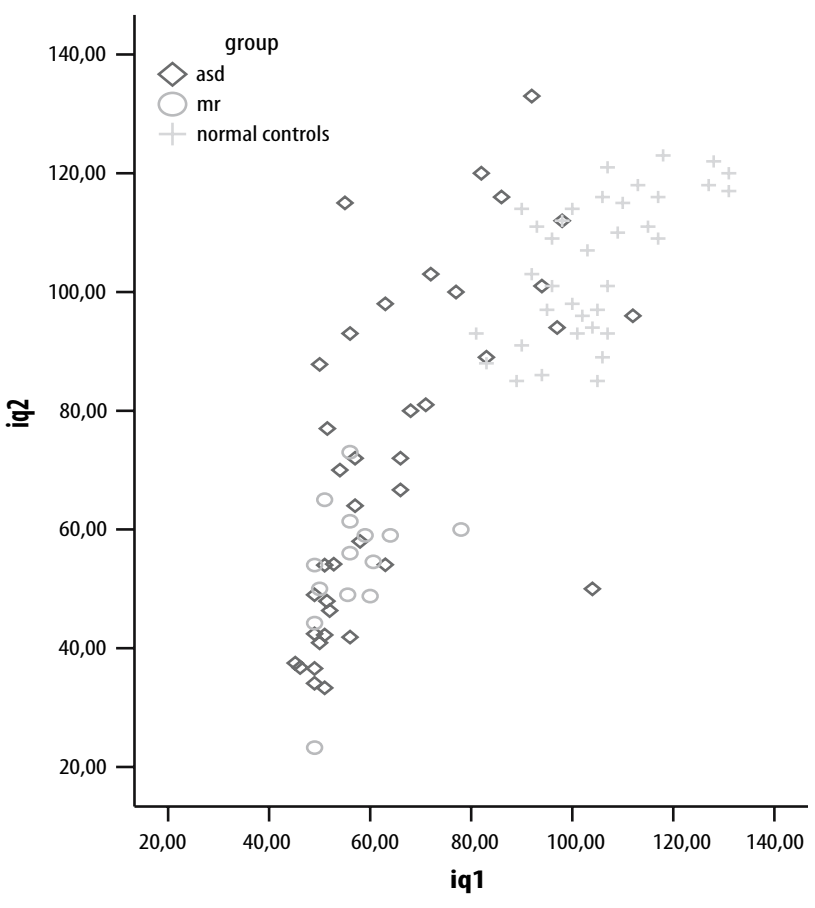

Fig. 1 Correlations between IQ score at T1 (X-axis) and IQ score at T2 (Y-axis), for the different diagnostic groups; ASD (autistic spectrum disorder); MR (mental retardation); and NC (normal controls)

$P \leq .01 ; N=89$ ). Correlations between IQ scores at T1 and T2 were high in the group of children diagnosed with ASD $(r=.65, P \leq .01 ; N=39)$ and similar to those of the NC group $(r=.63, P \leq .01 ; N=36)$. Correlations were low in the MR group $(r=.36$, $P=.21 ; N=14$ ) (see Fig. 1 ).

\section{IQ stability measured by average IQ change between $\mathrm{T} 1$ and $\mathrm{T} 2$}

IQ change was measured at a group level between $\mathrm{T} 1$ and T2. The average change in IQ scores was greatest in the ASD group $(M=6.78, \mathrm{SD}=21.55)$, in between in the MR group $(M=-2.57, \mathrm{SD}=$ $11.39)$, and lowest in the NC group $(M=-0.19$, $\mathrm{SD}=10.63$ ). The scores of the ASD group were variable, and there was evidence of heterogeneity of variance tested with Levene's statistic $(P=.002)$. The variance in IQ scores for the three subgroups is shown in Fig. 2.

\section{Individual IQ change between $\mathrm{T} 1$ and $\mathrm{T} 2$}

IQ change was also measured at individual level. As IQ is usually defined with confidence intervals, and gaining or losing some IQ points is clinically not significant, we considered a difference of at least 15

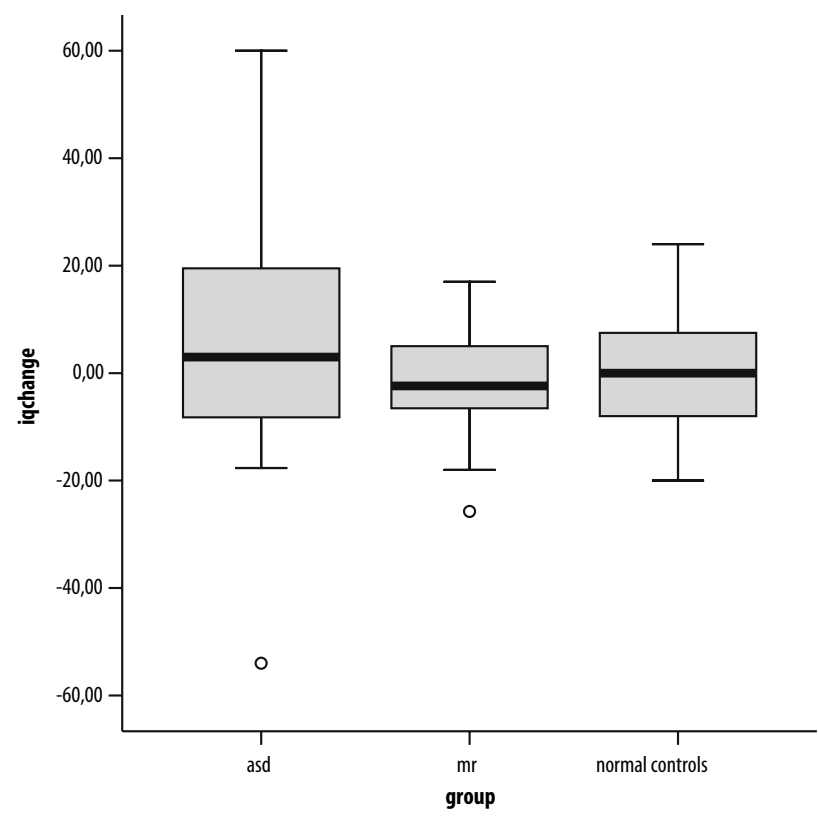

Fig. 2 Absolute IQ change between T1 and T2 at a group level. Two outliers can be identified: The child within the ASD group had average scores at first measurement however development came to a standstill at about this age resulting in a considerable IQ decrease. The child within the MR group was already low functioning at $\mathrm{T} 1$ but developed very slow between the measurements resulting in even lower IQ at T2

IQ points to be clinically relevant, because this difference equals one standard deviation from the mean of 100 , which is used in conventional cognitive tests. In the ASD group, the IQ score increased significantly in 12 children and decreased in 3 children. This increase was seen not only in children functioning within the (below) average range (5 of 12 children; $42 \%$ ), but also in children functioning within the MR range at T1 (7 of 27 children; 26\%). In the MR group, IQ scores increased in two children and decreased in one child; in the NC group the IQ scores increased in two children and decreased in two children (Fig. 3).

\section{Factors related to IQ increase}

The children with ASD with a clinically relevant increase in IQ score were compared with the children without such an increase. The ESAT score was lower in the children with a clinically relevant increase in IQ score, and these children had a significantly higher IQ score at T2 and better language skills, measured with the MSEL subscale 'Expressive Language'. The use of a particular cognitive test was not associated with the increase in IQ score. In 2 of 16 children with an increase in IQ score different cognitive tests were used at $\mathrm{T} 1$ and $\mathrm{T} 2$. 


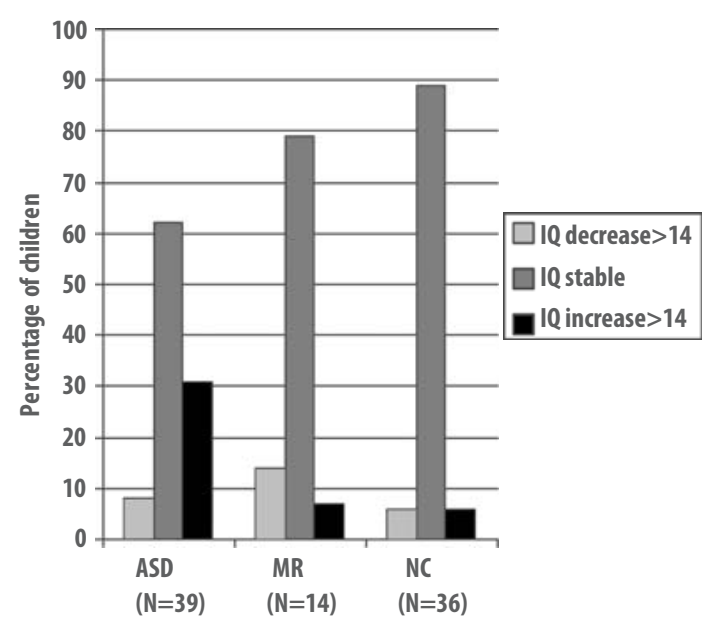

Fig. 3 Percentage of children diagnosed with ASD (autistic spectrum disorder), MR (mental retardation and NC (normal controls), in whom IQ increased ( $\uparrow$ at least 15 IQ points), decreased ( $\downarrow$ at least 15 points), or remained stable (↔ change less than $15 \mathrm{IQ}$ points)

\section{Discussion}

In this study, we report follow-up data on the cognitive functioning of 89 preschool children diagnosed with $\operatorname{ASD}(N=39)$, or MR $(N=14)$ and $\mathrm{NC}(N=36)$. Cognitive scores measured when the children were 2 years and 4 years were strongly correlated. This suggests that early cognitive testing of children with ASD is meaningful and that measuring cognitive functioning as part of the diagnostic process at early age is justified.

However, the IQ scores of about a third of the children diagnosed with ASD increased significantly between the two measurement times, whereas such an increase was detected in only a few children in the MR and NC groups. Few children showed a deterioration of intellectual functioning, and deterioration was not associated with any group in particular. Increase in IQ scores in children with ASD has been reported in other studies of preschool children [7, 32], and children between preschool years and early adolescence $[19,28]$.

We did not find higher functioning children in particular to show intellectual improvement $[7,19]$. Instead, IQ improved across the whole range of intellectual functioning (as measured at T1). Our data suggest that cognitive outcome of children with an IQ score in the MR range is dependent on the presence of ASD and is more favorable in children with MR and ASD. The uneven pattern of cognitive functioning found in most autistic individuals, some of whom show outstanding non-verbal skills, compared with the relatively evenly distribution of deficits found in most individuals diagnosed with MR without ASD may play a role. We did not find clear evidence, that the IQ improvement was associated with symptom severity, as was found by McGovern and Sigman [20], although we did find that the children who showed an IQ improvement had lower scores at the 14-item ESAT at T1.

The improvement in cognitive scores between 2 and 4 years of age may reflect the plasticity of the "young" brain and thus the potential for early learning and catch-up of intellectual functioning. However, these children need to be followed up for longer to determine whether the changes in IQ scores are meaningful and lasting. Moffit et al. [21] found IQ change to disappear after a third measurement in their sample of typically developing children of primary school age, and therefore considered the change negligible in amount, unreliably measured or both. A longer follow up would enable us to determine the effect of an increase in IQ score on outcome in adolescence or adulthood.

An increase in IQ score has been reported in several intervention studies $[15,16,27]$ and is sometimes claimed to be a result of intervention. Due to methodological problems, the actual impact of interventions has not been proven $[17,18]$. We found no association between the increase in IQ score and the intensity of the intervention; however our classification of intervention was rather rough and the type of treatment was not recorded.

\section{Conclusions}

The high correlation between cognitive scores measured 2 years apart in preschool children diagnosed with ASD suggests that measurements of cognitive function at this young age are valid. We found indications of both stability and change of IQ scores in preschoolers diagnosed with ASD. Therefore, caution is warranted when predicting future intellectual development on the basis of earlier measurements in very young children with developmental disorders. Our findings suggest that catch-up intellectual development occurs in some children diagnosed with ASD. To the best of our knowledge, this increase in IQ scores cannot be attributed to treatment effects.

\section{Limitations}

We used American normative data because normative Dutch data were not available when the study was performed. As the scores of the control group remained stable over the study period, we can cautiously assume that Dutch and American normative data are similar. Even if the normative data are different, the overall trend in cognitive scores we found in our study will probably be the same. 
A potential weakness of our study is the relatively short follow-up period of 2 years. Other studies investigated developmental trajectories over a much longer period, e.g. into adolescence [20] or adulthood [11]. More controlled and longer follow-up data for preschool children with ASD and other developmental disorders are needed to learn more about cognitive trajectories as well as factors influencing cognitive development.
Acknowledgments This study was supported by grants $940-38$ 045 and 940-38-014 (Chronic Disease Program), by grant 28.3000-2 of the Praeventiefonds-ZONMW, by the Netherlands Organisation for Scientific Research (NWO), by a grant from the Dutch Ministry of Health, Welfare and Culture, and by grants from Cure Autism Now, and the Korczak Foundation.

\section{References}

1. Achenbach TM (2000) Manual for the Child Behavior Checklist 1.5-5. Burlington, University of Vermont Department of Psychiatry

2. American Psychiatric Association (2000) Diagnostic and statistical manual of mental disorders (4th ed. text revision). Washington DC: American Psychiatric Association

3. Berument SK, Rutter M, Lord C, Pickles A, Bailey A (1999) Autism screening questionnaire: diagnostic validity. $\mathrm{Br} \mathrm{J}$ Psychiatry 175:444-451

4. Cox A, Klein K, Charman T, Baird G, Baron-Cohen S, Swettenham J, Wheelwright S, Drew A (1999) Autism Spectrum Disorders at 20 and 42 months of age: stability of clinical and ADI-R diagnosis. J Child Psychol Psychiatry 40:719-732

5. Dietz C, Swinkels SHN, Van Daalen E, Van Engeland H, Buitelaar JK (2006) Screening for Autistic Spectrum Disorders in children aged 14 to 15 months. II: population screening with the early screening of autistic traits questionnaire (ESAT): design and general findings. J Autism Dev Disord 36:713-722

6. Dilavore PC, Lord C, Rutter M (2000) The pre-linguistic autism diagnostic observation schedule. J Autism Dev Disord 25:355-379

7. Eaves LC, Ho HH (2004) The very early identification of autism: outcome to age $4 \frac{1}{2}-5$. Dev Med Child Neurol 34:367-378

8. Filipek PA, Accardo PJ, Baranek GT, Cook EH, Dawson G, Gordon B, et al. (1999) The screening and diagnosis of autistic spectrum disorders. J Autism Dev Disord 29:439-484

9. Fisch GS, Simensen RJ, Schroer RJ (2002) Longitudinal changes in cognitive and adaptive behavior scores in children and adolescents with the fragile X mutation or autism. J Autism Dev Disord 32:107-114

10. Howlin P, Asgharian A (1999) The diagnosis of autism and asperger syndrome: findings from a survey of 770 families. Dev Med Child Neurol 41:834839
11. Howlin P, Goode S, Hutton J, Rutter M (2004) Adult outcome for children with autism. J Child Psychol Psychiatry 45(2):212-229

12. Lord C (1995) Follow-up of two-yearolds referred for possible autism. J Child Psychol Psychiatry 36:1365-1382

13. Lord C, Rutter M, Le Couteur A (1994) Autism Diagnostic Interview-Revised. J Autism Dev Disord 24:659-686

14. Lord C, Risi S, DiLavore PS, Shulman C, Thurm A, Pickles A (2006) Autism from 2 to 9 years of age. Arch Gen Psychiatry 63:694-701

15. Lovaas OI (1987) Behavioral treatment and normal educational and intellectual functioning in young autistic children. Arch Gen Psychiatry 55:3-9

16. Lovaas OI (1993) The development of a treatment-research project for developmentally disabled and autistic children. J Appl Behav Anal 26:617-630

17. Magiati I, Howlin P (2001) Monitoring the progress of preschool children with autism enrolled in early intervention programmes. Autism 5(4):399-406

18. Matson JL (2007) Determining treatment outcome in early intervention programs for autism spectrum disorders: a critical analysis of measurement issues in learning based interventions. Research in developmental disabilities 28(2):207-218

19. Mayes SD, Calhoun SL (2003) Ability profiles in children with autism. Autism 6:65-80

20. McGovern CW, Sigman M (2005) Continuity and change form early childhood to adolescence in autism. J Child Psychol Psychiatry 46:401-408

21. Moffit TE, Caspi A, Harkness AR, Silva PA (1993) The natural history of change in intellectual performance: who changes? How much? Is it meaningful? J Child Psychol Psychiatry 34: 455-506

22. Mullen EM (1995) Mullen scales of early learning. Circle Pines MC, American Guidance Service

23. Neisworth JT, Bagnato SJ (2004) The mismeasure of young children: the authentic assessment alternative. Infants Young Child 17:198-212
24. Nordin V, Gillberg C (1998) The longterm course of autistic disorders: update on follow-up studies. Acta Psychiatr Scand 97(2):99-108

25. Rapin I (2003) Value and limitations of preschool cognitive tests, with an emphasis on longitudinal study of children on the autistic spectrum. Brain Dev 25:546-548

26. Schopler E, Reichler RJ, Bashford A, Lansing MD, Marcus LM (1990) Individualized assessment and treatment for autistic and developmentally disabled children Volume 1: psychoeducational profile-revised (pep-e) austin, Tex Pro-Ed

27. Sheinkopf SJ, Siegel B (1998) Homebased behavioural treatment of young children with autism. J Autism Dev Disord 28:15-23

28. Sigman M, Ruskin E, Arbeile S, Corona $\mathrm{R}$, Dissanayake C, Espinosa $\mathrm{M}$, et al. (1999) Continuity and change in the social competence of children with autism, Down syndrome, and developmental delays. Monogr Soc Res Child Dev 64:1-114

29. Sparrow SS, Balla DA, Cicchetti DV (1997) Vineland social-emotional early childhood scales manual. Circle Pines MC, American Guidance Service

30. Swinkels SHN, Dietz C, Van Daalen E, Kerkhof IHGM, Van Engeland H, Buitelaar JK (2006) Screening for Autistic Spectrum Disorders in children aged 14 to 15 months. I: the development of the Early Screening of Autistic Traits Questionnaire (ESAT). J Autism Dev Disord 36:723-732

31. Vohr BR, Carcia-Coll C, Oh W (1989) Language and neurodevelopmental outcome of low-birthweight infants at three years. Dev Med Child Neurol 31:582-590

32. Yang P, Jong Y, Hsu H, Chen C (2003) Preschool children with autism spectrum disorders in Taiwan: follow-up of cognitive assessment to early school age. Brain Dev 25:549-554 